\title{
The feasibility of computer-based prism adaptation to ameliorate neglect in sub-acute stroke patients admitted to a rehabilitation center
}

\author{
Miranda Smit ${ }^{1,2}$, Stefan Van der Stigchel ${ }^{2}$, Johanna M. A. Visser-Meily ${ }^{1,3}$, Mirjam Kouwenhoven ${ }^{3}$, \\ Anja L. H. Eijsackers ${ }^{3}$ and Tanja C. W. Nijboer ${ }^{1,2,3,4}$ * \\ Rudolf Magnus Institute of Neuroscience, Center of Excellence for Rehabilitation Medicine, University Medical Center Utrecht and De Hoogstraat, Utrecht, \\ Netherlands \\ ${ }^{2}$ Department of Experimental Psychology, Utrecht University, Utrecht, Netherlands \\ ${ }^{3}$ Rehabilitation Centre De Hoogstraat, Utrecht, Netherlands \\ ${ }^{4}$ Department of Neurology, University Medical Center Utrecht, Utrecht, Netherlands
}

\section{Edited by:}

Hauke R. Heekeren, Freie Universität

Berlin, Germany

\section{Reviewed by:}

Alessandro Farne, INSERM, France Sabrina Pitzalis, University of Rome

Foro Italico, Italy

\section{*Correspondence:}

Tanja C. W. Nijboer, Department of

Experimental Psychology, Helmholtz Institute, Utrecht University,

Heidelberglaan 2, 3584 CS Utrecht,

Netherlands

e-mail: t.c.w.nijboer@uu.nl
Introduction: There is wide interest in transferring paper-and-pencil tests to a computerbased setting, resulting in more precise recording of performance. Here, we investigated the feasibility of computer-based testing and computer-based prism adaptation (PA) to ameliorate neglect in sub-acute stroke patients admitted to a rehabilitation center.

Methods: Thirty-three neglect patients were included. PA was performed with a pair of goggles with wide-field point-to-point prismatic lenses inducing an ipsilesional optical shift of $10^{\circ}$. A variety of digitalized neuropsychological tests were performed using an interactive tablet immediately before and after PA.

Results: All 33 patients [mean age 60.36 (SD 13.30)], [mean days post-stroke 63.73 (SD 37.74)] were able to work with the tablet and to understand, perform, and complete the digitalized tests within the proposed time-frame, indicating that there is feasibility of computer-based assessment in this stage post-stroke. Analyses of the efficacy of PA indicated no significant change on any of the outcome measures, except time.

Discussion: In conclusion, there is feasibility of computer-based testing in such an early stage, which makes the computer-based setting a promising technique for evaluating more ecologically valid tasks. Secondly, the computer-based PA can be considered as a reliable procedure. We can conclude from our analysis, addressing the efficacy of PA, that the effectiveness of single session PA may not be sufficient to produce short-term effects on our static tasks. Further studies, however, need to be done to evaluate the computerbased efficacy with more ecologically valid assessments in an intensive double-blind, sham-controlled multiple PA treatment design.

Keywords: neglect, stroke, feasibility, efficacy, computer-based assessment, computer-based prism adaptation

\section{INTRODUCTION}

One of the major recent advances in neuropsychology is the use of computers during both screening and rehabilitation. There is wide interest in transferring paper-and-pencil tests to a computerbased setting (Schatz and Browndyke, 2002), resulting in a more detailed and precise recording of performance during screening and training (Rabuffetti et al., 2002; Chiba et al., 2006; Tsirlin et al., 2009) as well as enhanced consistency in testing across settings, making comparisons across patients more valid. Consequently, as the prism adaptation (PA) procedure has a fairly easy and repetitive design (see below), it is a good candidate for computer-based rehabilitation. Here, we investigate the feasibility of computerbased assessment and PA to ameliorate neglect in sub-acute stroke patients admitted to a rehabilitation center.

Neglect is a disabling disorder that frequently occurs after right hemisphere stroke (Bowen et al., 1999; Ringman et al., 2004). It refers to the failure to report, respond, or orient to stimuli on the contralesional side of space or body that cannot be accounted for by primary sensory or motor deficits (Halligan and Marshall, 1991; Robertson, 1999). Neglect is associated with poor functional recovery (Cherney et al., 2001; Jehkonen et al., 2006). Farne et al. (2004) found that whereas $43 \%$ of neglect patients demonstrated spontaneous recovery in the first 2 weeks, only $9 \%$ recovers completely. These findings concur with a recent study where patients were assessed several times during 1 year post-stroke (Nijboer et al., in press). In this study, spontaneous recovery of neglect appears to occur mainly during the first 12-14 weeks after stroke (Nijboer et al., in press) even though approximately $40 \%$ of the neglect patients do not fully recover and still show neglect on neuropsychological tests a year after stroke (Karnath et al., 2011; Rengachary et al., 2011; Nijboer et al., in press). Development of effective treatment techniques is therefore an important aim in 
neglect research, especially in the sub-acute phase, as the brain is primed to neurological recovery in the first 3 months post-stroke (Kwakkel et al., 2004; Murphy and Corbett, 2009).

One of the most widely investigated techniques to ameliorate neglect is PA (Rossetti et al., 1998; for overview, see Newport and Schenk, 2012). PA, originally proposed by Rossetti et al. (1998), is a promising experimental technique with strong therapeutic potential (Kerkhoff and Schenk, 2012). PA induces a proprioceptive shift in space by repetitive pointing to visual targets, resulting in a recalibration of the egocentric coordinate system. In other words, it creates a pointing bias in the opposite direction after prism removal and a contralesional shift in subjective body midline (Heilman et al., 1983; Saj and Vuilleumier, 2007). Positive effects of PA have been reported across many visuo-manual tasks in patients in the chronic phase, such as bisecting lines, line crossing, copy drawing (Saevarsson et al., 2010; Striemer and Danckert, 2010; Sarri et al., 2011), but also in more non-manual tasks, such as picture scanning, object-naming tasks and reading tasks (words and non-words) (Farne et al., 2002), and daily situations, such as wheelchair navigation (Rossetti et al., 1999; Watanabe and Amimoto, 2010) and postural control (Tilikete et al., 2001). The beneficial effects of PA have been reported to last $2 \mathrm{~h}$ (Rossetti et al., 1998) up to 1 week (Pisella et al., 2002; Dijkerman et al., 2004), and even up to 2 years (Nijboer et al., 2011).

Notwithstanding these promising results, evidence on feasibility and secondarily the efficacy of PA in the sub-acute stroke stage in a rehabilitation setting is relatively scarce. Nonetheless, it is important to identify an optimal or "critical period" for the optimal treatment response, keeping in mind that neurological recovery takes place in the first 3 months post-stroke (Kwakkel et al., 2004; Murphy and Corbett, 2009; Nijboer et al., in press). There are only a few randomized control trials that assessed the efficacy of PA exclusively in sub-acute (range 2-86 days) stroke patients (Nys et al., 2008; Turton et al., 2010; Mizuno et al., 2011). The effectiveness of PA in this stage remains equivocal, however. Whereas Mizuno et al. (2011) performed an intensive 2-week PA treatment and found improvement on the conventional Behavioral Inattention Test (BIT) and on a functional independence measure, Turton et al. (2010) did not find such an effect. The lack of efficacy in the latter study might be attributable to the use of $6^{\circ}$ goggles, which is a lesser degree of lateral displacement than that used in other studies (Barrett et al., 2012). Nys et al. (2008) only found short-term superiority in performance on the BIT, compared to placebo treatment. Clearly, the effectiveness of PA in the sub-acute stage post stroke needs further research.

To date, we do not know whether testing in general and computer-based assessment is too difficult or too time-consuming in this stage of syndrome since many previous studies were performed in the chronic stage. Therefore, our aim is to investigate the feasibility of computer-based assessment in a sub-acute stage post-stroke. Secondly, we combined the widely used PA procedure with a computer-based setting in order to investigate the feasibility of computer-based treatment (PA). Importantly, it is unknown whether a prismatic after-effect can be obtained with computerbased treatment. Furthermore, we gain insight in the adaptation procedure by means of more detailed and precise recordings of the pointing movements during the adaptation procedure as well as the magnitude of the after-effect. Lastly, we want to investigate the efficacy of a single session of computer-based PA on neuropsychological digitalized tests.

\section{METHODS}

\section{PARTICIPANTS}

In this study 33 stroke patients [mean age 60.36 (SD 13.30); mean days post-stroke 63.73 (SD 37.74)] with neglect (31 left visuospatial neglect) were included (see Table 1 for patient characteristics). All patients were admitted to rehabilitation center de Hoogstraat. Patients were included when they met the following criteria: (1) a brain lesion as revealed by CT or MRI; (2) presence of spatial neglect as assessed with a short screening including the Object Cancelation and Letter Cancelation (see Inclusion Based on Short Neuropsychological Screening below in this paragraph); (3) aged between 18 and 80 years; (4) able to understand and carry out the test instructions; (5) written or verbal informed consent and sufficient motivation to participate.

All patients received multidisciplinary standard stroke care and treatment and participating in the study did not interfere with daily routines. Additionally, all patients received visual scan training, which was the current intervention used for rehabilitation of neglect in this rehabilitation center.

\section{Inclusion based on short neuropsychological screening}

The short screening that took place prior to the inclusion of the present study was part of the standard stroke care. At this level, severity of neglect was evaluated on the bases of the standard outcome measures of the Object Cancelation and the Letter Cancelation (number of omissions). The average number of omissions was 7.21 $(\mathrm{SD}=8.39)$ for the Object Cancelation and 7.11

Table 1 | Demographical and stroke characteristics of the included patients.

\begin{tabular}{ll}
\hline Clinical variables & Included pat \\
\hline Group size & 33 \\
Age (years) & $60(13.30)$ \\
Gender (male) & $57.58 \%$ \\
Stroke characteristics & \\
Days post-stroke & $63.73(37.74)$ \\
Hemisphere of stroke $(R)$ & $90.91 \%$ \\
Unilateral & $96.97 \%$ \\
Type of stroke & \\
$\quad$ Cortical ischemia & $63.64 \%$ \\
Subcortical ischemia & $3.03 \%$ \\
Intracerebral hemorrhage & $30.30 \%$ \\
Other & \\
Barthel index $(n=28)$ & $3.03 \%$ \\
Motricity index arm $(n=23)$ & $12.07(5.77)$ \\
Motricity index leg $(n=23)$ & $57.43(41.38)$ \\
MMSE ( $n=25)$ & $67.43(36.75)$ \\
Hemianopia & $25.54(4.29)$ \\
& $39.39 \%$
\end{tabular}

MMSE, mini mental state exam; *ischemia due to acute disseminated encephalomyelitis. 
$(S D=5.80)$ for the Letter Cancelation. It should be noted that only 15 patients of our total sample performed the Letter Cancelation in the screening. When evaluating the level of lateralized impairment, we calculated the asymmetry in the number of omitted items. For the Object Cancelation, 27 patients (out of 33) showed an asymmetry in omitted items in the range from 0 to $10(M=2.44 ; \mathrm{SD}=2.39)$ and 6 patients had an asymmetry score between 15 and $30(M=19.33$; SD 3.78). For the Letter Cancelation test (LC), 16 (out of 18) patients displayed an lateralized deficit between 0 and $10(M=3.63$; SD 3.07) and 2 patients had an asymmetry score between 10 and $15(M=14.00$; SD 1.41).

\section{APPARATUS}

Both the PA procedure and the neuropsychological tests were done using a 22-inch interactive WACOM (PL2200) tablet screen $(1920 \times 1080)$, with a screen size of $477.64 \mathrm{~mm} \times 268.11 \mathrm{~mm}$. The tablet includes a widescreen display (luminance: $200 \mathrm{~cd} / \mathrm{m}^{2}$ ) and full HD resolution $(0.01 \mathrm{~mm} /$ point $)$ and has a screen refresh rate of $5 \mathrm{~ms}$. The display offers a large working area and provides good spatial $( \pm 0.01 \mathrm{~mm} /$ point$)$ and temporal resolution (133 points/s, max).

Patients had to respond to stimuli by drawing on or pointing at the screen with a digital stylus. We used an electromagnetic resonance method to record patients' performance of the stylus. DiagnoseIS (developed by Metrisquare, Netherlands) was used to program the neglect screening tests (e.g., Cancelation tests). The tablet was driven by a laptop in order to monitor stimuli and patients performance on the experimenter's laptop. During performance the tablet screen was oriented horizontally and slightly tilted $\left(18^{\circ}\right)$ with an adjustable stand.

\section{DIGITALIZED PRISM ADAPTATION PROCEDURE}

The PA procedure was adapted from Rossetti et al. (1998) and was performed with a pair of goggles fitted with wide-field point-to-point prismatic lenses, inducing a rightward optical shift of $10^{\circ}$.

The distance between the visual stimuli and the body midline was approximately $65 \mathrm{~cm}$. Patients were presented with three visual targets (red, yellow, blue) on a horizontal axis. The left and right visual targets were both $11.5 \mathrm{~cm}$ away from a central visual stimulus. Exposure consisted of 100 fast repetitive pointing movements. Half of the pointing movements were made to the left visual target, the other half were made to the right visual target. Patients were occasionally instructed to point to the central visual stimulus when pointing appeared to become a routine. These additional pointing movements prevented automatic pointing in a sequence of motor acts to either the left or the right target. When patients experienced difficulties in distinguishing between left and right, the color of the visual stimulus was used. Whenever the patient touched the tablet screen with the digital stylus, $x$ and $y$ coordinates and timing data were recorded. Error reduction was achieved when patients hit the target. Patients pointing performance was only and immediately presented at the laptop of the experimenter, allowing the experimenter to monitor the accuracy of the pointing movements online.

After the adaptation phase (e.g., repetitive pointing), prisms were withdrawn and the after-effect was measured.
Conventionally, the strength of the adaptation can be obtained by measuring the spatial deviation from a target stimulus. During the repetitive pointing movements (the adaptation phase), visuomotor corrections toward the contralateral side in order to point to the target as accurate as possible, are executed. Thus, when prisms are removed, the spatial deviation will be in the opposite direction of the visual displacement imposed by the prism glasses, a phenomenon known as the after-effect. In our sample we used the central target to measure the after-effect. Here, after prism removal, patients were instructed to look carefully at the central visual target. After a few seconds they were instructed to point with the digital stylus at the central target, with eyes closed to prevent online adjustments. Again, patients did not get feedback about the landing position of the digital stylus, which was only shown at the experimenter's laptop. For after-effects, the mean error displacement from the central stimulus was calculated and should have been at least $3 \mathrm{~cm}$, otherwise the PA procedure was continued.

\section{STIMULI, TESTS, AND PROCEDURE}

All measurements were conducted in a sound-attenuated room. Patients were seated as comfortable as possible, in upright position in front of the tablet. All tests were done using the tablet. These tests were done prior and immediately after PA in the same order if possible. The whole test procedure lasted for an hour and patients were allowed to have a small break when needed prior or after the PA procedure.

In an Object Cancelation test (OC), patients were presented with 54 targets, and 75 distractors. Patients had to cross out all the targets. Patients were given feedback; they could see their own performance; e.g., see the stripe of the digital stylus through the canceled items. Outcome measurements were the total number of omissions, total time for test completion, search time in the ipsilesional and contralesional field and the horizontal and vertical Center of Cancelation $(\mathrm{CoC})$. The $\mathrm{CoC}$ is an indicative measure of severity of neglect, since it obtains information of both the number of omissions and the location of canceled items. Generally, a positive CoC-score $(+)$ indicates that the mean horizontal location of the canceled items is at the right side of the stimulus sheet, e.g., indicating lateralized deficits on the far left and vice versa. A CoC-score toward zero means a more symmetrical spatial error distribution. Calculations for the CoC were adapted from Rorden and Karnath (2010). Additionally, the same method was applied for the number and spatial distribution of perseverations.

The Letter Cancelation test (LC) consisted of 5 rows of 34 random letters (170 letters in total). Patients were instructed to cancel the target letters, which were randomly placed between the distractor letters. Outcome measures were the total number of omissions, total time for test completion, search time in the ipsilesional and contralesional field and the horizontal CoC (Rorden and Karnath, 2010).

In a Line bisection test (LB), patients were presented with three horizontal lines $(31 \mathrm{~cm}$ in length and $1 \mathrm{~mm}$ in width). The lines were outlined in a staircase fashion and patients had to indicate (upper to low) the true center of each line. This test was performed twice. Outcome measurement was the total deviation of the true midpoint for each line and total time for test completion. 


\section{ANALYSES}

In the first part of the result section qualitative information about the feasibility of computer-based testing and computer-based treatment will be addressed. Moreover, reasons of exclusion will be specified.

In the second part, analyses of the efficacy of PA will be discussed. Regarding the cancelation tests; 27 and 26 patients were included in the OC and LC, respectively (see below for reason of exclusion). For both tasks paired sample $t$-tests were performed between pre- and post-test for total number of omissions, total time for test completion, and the search-times on both the ipsilesional and contralesional side. Moreover, the mean $\mathrm{CoC}$ were calculated for both the cancelation tasks. The mean Center of Perseveration was only calculated for the OC, due to a small amount of perseverations in the LC. Paired samples $t$-tests were performed between the pre-horizontal and vertical $\mathrm{CoC} / \mathrm{CoP}$ and the post-horizontal and vertical CoC/CoP.

For the LB, 27 patients were included (see below for reason of exclusion). Paired samples $t$-tests were performed between presession and post-session for the mean deviation of the true center (i.e., mean line $1^{\mathrm{a}}$ pre-test and line $1^{\mathrm{b}}$ pre-test versus mean line $1^{\mathrm{a}}$ post-test and line $1^{\mathrm{b}}$ post-test, likewise for line 2 and 3 ) and total time for test completion. For all tests, since we had both left and right neglect patients in our sample, we made "contralesional" and "ipsilesional" classifications in order to consider them as one group. A two-tailed significance level of 0.05 was used. Results of the efficacy of PA on the digitalized tests are outlined in Table 2.

\section{RESULTS}

\section{FEASIBILITY OF COMPUTER-BASED TESTING}

First, all 33 patients were capable to respond to stimuli by drawing, canceling on, or pointing at the screen with a digital stylus, indicating that computer-based testing was feasible. Second, considering the overall feasibility of performing tests in such an early stage post-stroke we observed that, all but one patient, performed the pre- and post OC and LB; that particular patient did not complete the post-test (as well for the LC) due to emotional factors. For the LC, 31 patients performed the pre- and post-test. One patient did not perform the pre- and post LC due to a language barrier and illiteracy. Third, patients were able to complete test performance within the proposed time-frame. The total duration of performing all digitalized tests, pre and post, was approximately $6.7 \mathrm{~min}$, which indicates that after the verbal instruction was given, patients worked continuously on the digitalized tasks, meaning that test instructions were understood quickly. The total duration of the PA (first pointing movement till after-effect) was, on average, $8.4 \mathrm{~min}$.

\section{FEASIBILITY OF COMPUTER-BASED PA IN SUB-ACUTE STROKE PATIENTS}

Generally, patients were able to perform the PA procedure, e.g., pointing at the screen with a digital stylus, on a computer. However, for some patients the PA procedure (repetitive pointing movements) was sometimes strenuous. Four patients experienced difficulties distinguishing left from right. Two out of four of these patients preferred color naming over left-right responses.
Additionally, one patient experienced a headache while pointing. This became less when the adaptation procedure proceeded. Due to repetitive pointing one patient experienced exhaustion of the right arm. For another patient, working with the digital stylus became difficult as a result of rheumatic problems.

Additionally, a Pearson correlation coefficient was performed in order to assess whether a relationship existed between neglect severity at baseline and the magnitude of the error displacement. Neglect severity was assessed with the "asymmetry score" of the OC from the neuropsychological screening, see participant section. However, Pearson correlation coefficient revealed no significant relationship between the magnitude of the error displacement and the asymmetry score in the OC, $r=0.132, n=33, p=0.463$. This indicates that neglect severity was not associated with the level of adaptation.

To recall, the strength of the adaptation was obtained by measuring the spatial deviation (in $\mathrm{cm}$ ) from the central stimulus, which is called the after-effect phenomenon (see Digitalized Prism Adaptation Procedure in Methods section). Regarding the magnitude of the after-effect, the mean error displacement from the center target of all the 33 patients was $4.12 \mathrm{~cm}$ (SD 2.00) with a minimum displacement of 0.38 and a maximum displacement of 7.24 , indicating that most, but not all patients, adapted well to the prism procedure (see Figure 1). For five patients the adaptation procedure was continued, and after a second adaptation phase, these patients still showed a minor error displacement $(M=1.26$; $\mathrm{SD}=0.81$ ). These patients were not included in the analyses on the efficacy of the digitalized neuropsychological tests (see below). One patient whom had also a small after-effect was not secondly adapted due to emotional factors (see above).

\section{EXCLUDED PATIENTS}

In sum, six patients were excluded from the overall analyses in the LB and OC. For the LC, seven patients were excluded.

\section{RECORDINGS OF THE POINTING MOVEMENTS DURING THE ADAPTATION PROCEDURE}

Recordings of the pointing movements revealed that the error displacement was the largest at the first five pointing movements

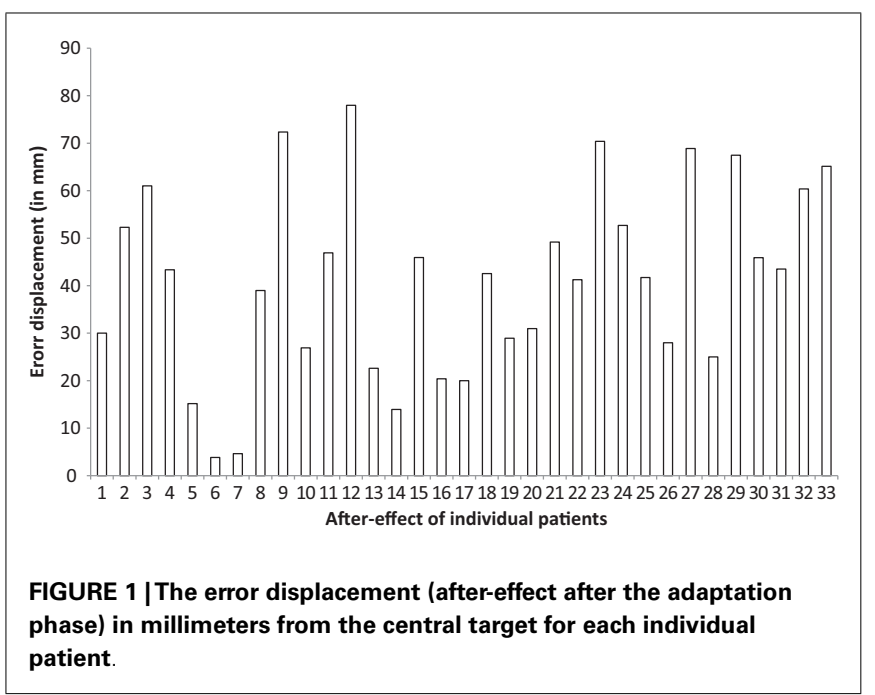


(see Figures 2 and 3). Thereafter the error displacement became relatively stable and patients become fairly accurate in pointing to either the left, right, or central target. This indicates that the process of recalibrating the new egocentric coordinate system sets in rapidly. Note that the absolute center of each target was used as a referent and not the whole target. In this regard, patients could have hit the target (i.e., error reduction was achieved), but not the true center $(x, y$ coordinate $)$ of that target.

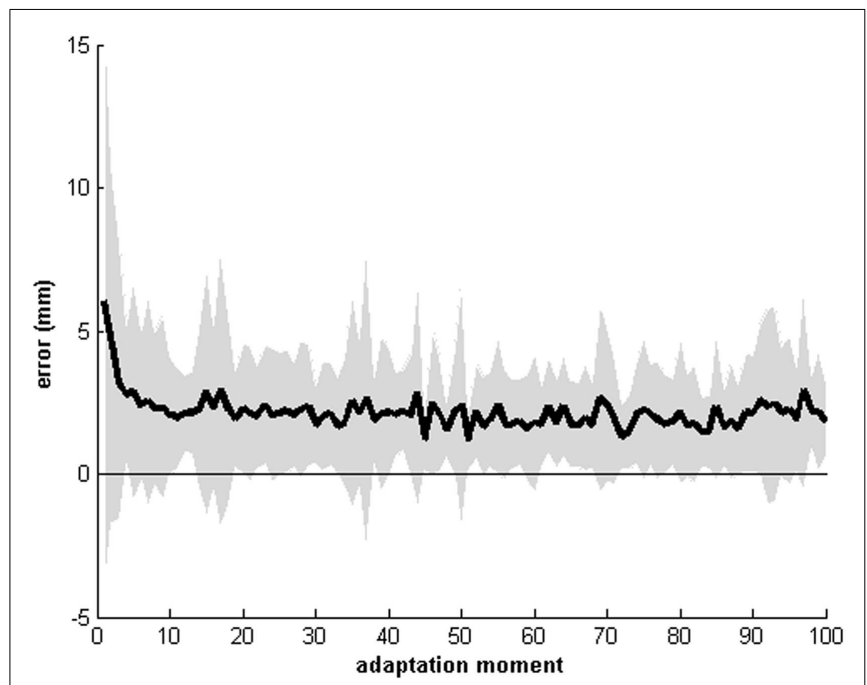

FIGURE 2 | Mean recordings of the pointing movements of all 33 patients for the first $\mathbf{1 0 0}$ pointing movements. The horizontal axis displays the moment of pointing (0 till 100), the vertical axis displays the error displacement of either the right, left, or central target. Shaded area indicates the mean standard deviation. Note that the absolute center of each target ( $x, y$ coordinate) was used as the referent.

\section{EFFICACY OF A SINGLE SESSION OF COMPUTER-BASED PA}

Analyses of the efficacy of PA did not reveal significant changes in deviation from the actual center on the line bisection nor in number of omissions on the cancelation tests. Additionally, no significant shift in the location of the omissions and perseverations was found ( $\mathrm{CoC}$ and $\mathrm{CoP}$ outcome measures). The total time taken to complete the test improved significantly after PA for the OC, LC, as well as the LB. Results of the digitalized tests are outlined in Table 2.

Regarding time for test completion, all tests were performed significantly faster (total time) after PA, see Table 2. Additionally, when addressing the search-times (Table 2) on either the ipsilesional as well as the contralesional side of the stimulus-sheets presented on the tablet, patients performed significantly faster post PA in the OC. For the LC this was only faster on the contralesional side post PA. Search-times were based on the search-times within one field, and could reflect either searching a target but also recursively exploring one side due to perseveratory behavior.

\section{GENERAL DISCUSSION}

The aim of the current study was to (1) investigate the feasibility of computer-based testing (2) and computer-based treatment in sub-acute stroke patients; (3) gain insight in the adaptation procedure by means of more detailed and precise recordings of pointing movements; and (4) investigate efficacy of a single session of computer-based PA on neuropsychological digitalized tests. Regarding the feasibility of computer-based neuropsychological assessment, all of the included patients were able to work with the tablet and to understand and perform the digitalized tests within the proposed time-frame. This indicates that there is feasibility of computer-based testing in an early stage post-stroke. Although, using this type of tablet and stylus was indeed feasible in our sample, it should be noted that

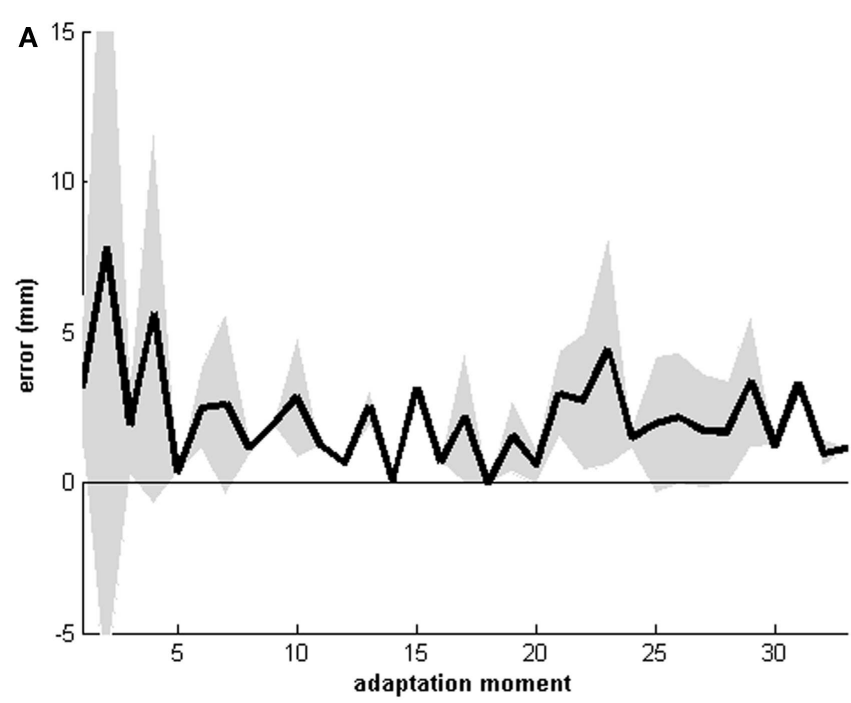

FIGURE 3 | Mean recordings of the pointing movements of all 33 patients for $\mathbf{3 0}$ pointing movements for either the "right" $(A)$ and the "left" (B) target. The horizontal axis displays the moment of pointing (0 till

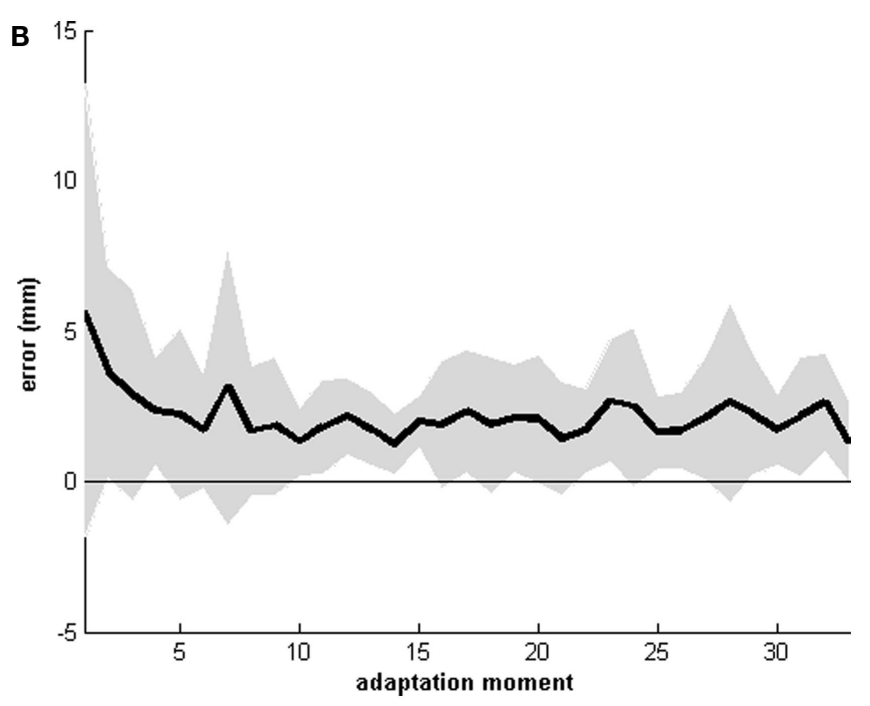

30), the vertical axis displays the error displacement from the right (A) or the left (B) target. Shaded area indicates the mean standard deviation. Note that the absolute center of the target ( $x, y$ coordinate) was used as the referent. 
Table 2 | Mean results of the digitalized pre-test and post-test.

\begin{tabular}{|c|c|c|c|c|c|}
\hline Test & $N$ & Outcome measure & Mean pre-test & Mean post-test & Statistics \\
\hline \multirow[t]{9}{*}{$\mathrm{OC}$} & 27 & Omissions total & $4.48(6.44)$ & $5.37(6.80)$ & $t(26)=-1.464, p=0.155$ \\
\hline & 27 & $\mathrm{CoC}-x$ & $0.04(0.13)$ & $0.05(0.13)$ & $t(26)=-0.417, p=0.680$ \\
\hline & & CoC-y & $0.00(0.02)$ & $0.00(0.03)$ & $t(26)=-0.910, p=0.371$ \\
\hline & 27 & Perseverations total & $7.04(10.87)$ & $6.52(11.83)$ & $t(26)=0.517, p=0.610$ \\
\hline & 27 & CoP-x & $0.08(0.30)$ & $0.06(0.41)$ & $t(26)=0.265, p=0.793$ \\
\hline & & CoP-y & $0.08(0.29)$ & $0.06(0.36)$ & $t(26)=0.232, p=0.818$ \\
\hline & $25^{1}$ & Total time in sec & $99.16(40.85)$ & $84.00(37.21)$ & $t(24)=3.318, p=0.003^{* *}$ \\
\hline & & Time contralesional & $32.40(18.38)$ & $25.80(16.05)$ & $t(24)=3.234, p=0.004^{* *}$ \\
\hline & & Time ipsilesional & $33.70(16.02)$ & $26.80(12.23)$ & $t(24)=4.082, p<0.0001^{* * *}$ \\
\hline \multirow[t]{6}{*}{ LC } & 26 & Omissions total & $6.23(7.00)$ & $5.00(5.69)$ & $t(25)=1.786, p=0.086$ \\
\hline & 26 & CoC-x & $0.06(0.16)$ & $0.04(0.14)$ & $t(25)=1.126, p=0.271$ \\
\hline & & CoC-y & $0.00(0.04)$ & $0.00(0.03)$ & $t(25)=0.088, p=0.931$ \\
\hline & $25^{1}$ & Total time in sec & $106.14(49.63)$ & 93.09 (31.73) & $t(24)=2.394, p=0.025^{*}$ \\
\hline & & Time contralesional & $25.52(11.78)$ & $22.85(11.28)$ & $t(24)=2.357, p=0.027^{*}$ \\
\hline & & Time ipsilesional & $28.54(10.03)$ & $27.93(9.89)$ & $t(24)=0.422, p=0.677$ \\
\hline \multirow[t]{4}{*}{$\mathrm{LB}(\mathrm{mm})$} & 27 & Deviation line $1^{a} 1^{b}$ & -1.97 (18.30) & $-1.81(12.82)$ & $t(26)=-0.061, p=0.952$ \\
\hline & & Deviation line $2^{a} 2^{b}$ & $-9.30(21.68)$ & $-8.77(19.34)$ & $t(26)=-0.268, p=0.791$ \\
\hline & & Deviation line $3^{a} 3^{b}$ & $-17.75(28.13)$ & $-23.78(29.61)$ & $t(26)=1.672, p=0.106$ \\
\hline & & Total time & $10.19(6.42)$ & $7.94(4.34)$ & $t(26)=2.458, p=0.021^{*}$ \\
\hline
\end{tabular}

Standard deviations are given in parenthesis. OC, Object Cancelation; LC, Letter Cancelation; CoC, Center of Cancelation; CoP, center of perseveration; LB, Line

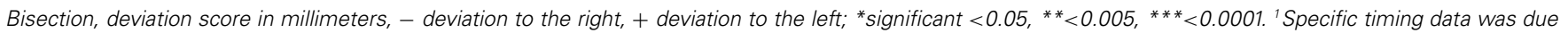
to a synchronization error not stored for two patients in the OC, and one patient in the LC.

our sample was relatively young and suffered from relatively mild neglect on neuropsychological neglect screening tests. Moreover, our sample rehabilitated with the intention to reintegrate in society as soon as possible. One might assume that these patients display fewer problems (both physically and mentally) than long stay stroke patients whom live in nursing homes. Our sample may thus not be representative for the overall stroke population. In this regard, a finger activated tablet might be a more appropriate solution in chronic patients or during bedside testing.

Not only can computer-based testing improve the traditional paper-and-pencil assessment of neglect by means of more precise and detailed recordings (Rabuffetti et al., 2002; Chiba et al., 2006; Tsirlin et al., 2009), it also holds opportunities in developing more ecologically valid tests. For instance, daily situations are far more dynamic and require fast responses in order to avoid obstacles in complex environments. We suggest that dynamic tests (e.g., ecologically based) increases the attentional load similar to daily life and will be more sensitive in detecting the "real level of neglect" (Tsirlin et al., 2009). This is impossible with the traditional paper-and-pencil tests.

In addition, the computer-based treatment can be considered as a reliable tool in performing PA, since most patients adapted well to the procedure, as quantified with the magnitude of the after-effects. These after-effects were comparable with our traditional (paper and pencil) PA after-effects (Nijboer et al., 2008, 2010, 2011; Bultitude et al., 2013). Moreover, detailed recordings of the pointing movements revealed that, on average, the error displacement was the largest for the first five pointing movements.
Thereafter the error displacement was relatively stable and patients became fairly accurate in pointing to either the left, right, or central target. This implies that the process of recalibrating a new egocentric coordinate system sets in rapidly. Combining computer-based treatment with computer-based ecologically valid assessments in the sub-acute stage seems like a promising thought in identifying a "critical period" for the optimal treatment response with more detailed measures, especially when neurological recovery takes place in the first 3 months post-stroke (Kwakkel et al., 2004; Murphy and Corbett, 2009; Nijboer et al., in press).

Analyses of the efficacy of PA indicated no significant change on any of the outcome measures, except time. However, our sample as a group showed only mild visual neglect at baseline in especially the cancelation tasks. In this regard, there was less room for further improvement. Second, it is likely that concurrent compensation training already changed the scanning strategy in these neglect patients and that one session of PA does not further enhance attentional processing. In addition, since our tests were statically presented at the tablet till task-completion, patients could apply their in-hospital learned cognitive strategy (top-down scanning strategies toward contralesional stimuli), which could have masked their real level of neglect. It would have been interesting to investigate differences in feasibility (pointing movements) and efficacy in both left- and right-sided neglect. The sample size of especially the group of right-sided neglect patients (2) was too small, however, to statistically compare the efficacy of single session PA between those two groups.

Moreover, our design was not fit to fully evaluate effects of PA. We did not use a control-group to counteract learning- and/or 
motivational-effects. Although we lacked an effect of PA on the digitalized tasks, we do not know whether PA had no effect at all. Subtle treatment effects could be overruled by fatigue at the end of the test-session, since post-stroke fatigue is common (De Groot et al., 2003; Lerdal et al., 2009). Furthermore, one session may be insufficient to produce long-term and even shortterm effects, which is in line with the conclusion in a recent review (Schenk and Karnath, 2012). However, generalizable and stable improvement of PA was found when using an intensive treatment program with multiple sessions (10 or more) of PA (Frassinetti et al., 2002; Serino et al., 2009; Mizuno et al., 2011). In addition, we do not know whether a computer-based setting influenced test performance either positively or negatively. It is possible that patients were more alert at the start of the novel test-situation (with a tablet) and became less alert when the computer-based setting became more familiar. In order to disentangle treatment effects from motivational effects and to

\section{REFERENCES}

Barrett, A. M., Goedert, K. M., and Basso, J. C. (2012). Prism adaptation for spatial neglect after stroke: translational practice gaps. Nat. Rev. Neurol. 8, 567-577. doi:10.1038/nrneurol.2012.170

Bowen, A., McKenna, K., and Tallis, R. C. (1999). Reasons for variability in the reported rate of occurrence of unilateral spatial neglect after stroke. Stroke 30, 1196-1202. doi:10.1161/01.STR.30.6.1196

Bultitude, J. H., Van der Stigchel, S., and Nijboer, T. C. (2013). Prism adaptation alters spatial remapping in healthy individuals: evidence from double-step saccades. Cortex 49, 759-770. doi:10.1016/j.cortex.2012.01.008

Cherney, L. R., Halper, A. S., Kwasnica, C. M., Harvey, R. L., and Zhang, M. (2001). Recovery of functional status after right hemisphere stroke: relationship with unilateral neglect. Arch. Phys. Med. Rehabil. 82, 322-328. doi:10.1053/apmr.2001. 21511

Chiba, Y., Yamaguchi, A., and Eto, F. (2006). Assessment of sensory neglect: a study using moving images. Neuropsychol. Rehabil. 16, 641-652. doi:10.1080/09602010543000073

De Groot, M. H., Phillips, S. J., and Eskes, G. A. (2003). Fatigue associated with stroke and other neurologic conditions: implications for stroke rehabilitation. Arch. Phys. Med. Rehabil. 84, 1714-1720. doi:10.1053/S0003-9993(03) 00346-0

Dijkerman, H. C., Webeling, M., ter Wal, J. M., Groet, E., and van Zandvoort, M. J. (2004). A long-lasting improvement of somatosensory function after prism adaptation, a case study. Neuropsychologia 42, 1697-1702. doi:10.1016/ j.neuropsychologia.2004.04.004

Farne, A., Buxbaum, L. J., Ferraro, M., Frassinetti, F., Whyte, J., Veramonti, T., et al. (2004). Patterns of spontaneous recovery of neglect and associated disorders in acute right brain-damaged patients. J. Neurol. Neurosurg. Psychiatr. 75, 1401-1410. doi:10.1136/jnnp.2002.003095

Farne, A., Rossetti, Y., Toniolo, S., and Ladavas, E. (2002). Ameliorating neglect with prism adaptation: visuo-manual and visuoverbal measures. Neuropsychologia 40, 718-729. doi:10.1016/S00283932(01)00186-5

Frassinetti, F., Angeli, V., Meneghello, F., Avanzi, S., and Ladavas, E. (2002). Long-lasting amelioration of visuospatial neglect by prism adaptation. Brain 125(Pt 3), 608-623. doi:10.1093/brain/awf056

Halligan, P. W., and Marshall, J. C. (1991). Spatial compression in visual neglect: a case study. Cortex 27, 623-629. doi:10.1016/S00109452(13)80011-1

Heilman, K. M., Bowers, D., and Watson, R. T. (1983). Performance on hemispatial pointing task by patients with neglect syndrome. Neurology 33, 661-664. doi:10.1212/WNL.33.5.661

Jehkonen, M., Laihosalo, M., and Kettunen, J. E. (2006). Impact of neglect on functional outcome after stroke: a review of methodological issues and recent research findings. Restor. Neurol. Neurosci. 24, 209-215.

Karnath, H. O., Rennig, J., Johannsen, L., and Rorden, C. (2011). The anatomy underlying acute versus chronic spatial neglect: a

control for a computer-based setting, a sham-controlled design is necessary.

In conclusion, there is feasibility of computer-based testing in such an early stage, which makes the computer-based setting a promising technique for evaluating more ecologically valid tasks. Secondly, the computer-based PA can be considered as a reliable procedure. We can conclude from our analysis, addressing the efficacy of PA, that the effectiveness of single session PA may not be sufficient to produce short-term effects on our static tasks. Further studies need to be done to evaluate the computer-based efficacy with more ecologically valid assessments in an intensive double-blind, sham-controlled multiple PA treatment design.

\section{ACKNOWLEDGMENTS}

This research was supported by the grant 451-10-013 from the Netherlands Organization for Scientific Research (NWO) to Tanja C. W. Nijboer.

longitudinal study. Brain 134(Pt 3), 903-912. doi:10.1093/brain/awq355

Kerkhoff, G., and Schenk, T. (2012). Rehabilitation of neglect: an update. Neuropsychologia 50, 1072-1079. doi:10.1016/j

Kwakkel, G., Kollen, B., and Lindeman, E. (2004). Understanding the pattern of functional recovery after stroke: facts and theories. Restor. Neurol. Neurosci. 22, 281-299.

Lerdal, A., Bakken, L. N., Kouwenhoven, S. E., Pedersen, G., Kirkevold, M. Finset, A., et al. (2009). Poststroke fatigue - a review. J. Pain Symptom. Manage. 38, 928-949. doi:10.1016/ j.jpainsymman.2009.04.028

Mizuno, K., Tsuji, T., Takebayashi, T., Fujiwara, T., Hase, K., and Liu, M. (2011). Prism adaptation therapy enhances rehabilitation of stroke patients with unilateral spatial neglect: a randomized, controlled trial. Neurorehabil. Neural Repair 25, 711-720. doi:10.1177/1545968311407516

Murphy, T. H., and Corbett, D. (2009). Plasticity during stroke recovery: from synapse to behaviour. Nat. Rev. Neurosci. 10, 861-872. doi:10.1038/nrn2735

Newport, R., and Schenk, T. (2012). Prisms and neglect: what have we learned? Neuropsychologia 50, 1080-1091. doi:10.1016/ j.neuropsychologia.2012.01.023

Nijboer, T., Vree, A., Dijkerman, C., and Van der Stigchel, S. (2010). Prism adaptation influences perception but not attention: evidence from antisaccades. Neuroreport 21, 386-389. doi: 10.1097/WNR.0b013e328337f95f

Nijboer, T. C., Kollen, B. J., and Kwakkel, G. (in press). Time course of visuospatial neglect early after stroke: a longitudinal cohort study. Cortex doi:10.1016/j.cortex.2012.11.006. [Epub ahead of print].

Nijboer, T. C., McIntosh, R. D., Nys, G. M., Dijkerman, H. C., and Milner, A. D. (2008). Prism adaptation improves voluntary but not automatic orienting in neglect. Neuroreport 19, 293-298. doi:10. 1097/WNR.0b013e3282f4cb67

Nijboer, T. C., Nys, G. M., van der Smagt, M. J., van der Stigchel, S., and Dijkerman, H. C. (2011). Repetitive long-term prism adaptation permanently improves the detection of contralesional visual stimuli in a patient with chronic neglect. Cortex 47, 734-740. doi:10.1016/j.cortex.2010.07.003

Nys, G. M., de Haan, E. H., Kunneman, A., de Kort, P. L., and Dijkerman, H. C. (2008). Acute neglect rehabilitation using repetitive prism adaptation: a randomized placebocontrolled trial. Restor. Neurol. Neurosii. 26, 1-12.

Pisella, L., Rode, G., Farne, A., Boisson, D., and Rossetti, Y. (2002). Dissociated long lasting improvements of straight-ahead pointing and line bisection tasks in two hemineglect patients. Neuropsychologia 40, 327-334. doi:10.1016/S00283932(01)00107-5

Rabuffetti, M., Ferrarin, M., Spadone, R., Pellegatta, D., Gentileschi, V., Vallar, G., et al. (2002). Touch-screen system for assessing visuo-motor exploratory skills in neuropsychological disorders of spatial cognition. Med. Biol. Eng. Comput. 40, 675-686.

Rengachary, J., He, B. J., Shulman, G. L., and Corbetta, M. (2011). A behavioral analysis of spatial neglect and its recovery after stroke. Front. Hum. Neurosci. 5:29. doi:10.3389/fnhum.2011.00029 
Ringman, J. M., Saver, J. L., Woolson, R. F., Clarke, W. R., and Adams, H. P. (2004). Frequency, risk factors, anatomy, and course of unilateral neglect in an acute stroke cohort. Neurology 63, 468-474. doi:10.1212/01.WNL.0000133011. 10689.CE

Robertson, I. H. (1999). Cognitive rehabilitation: attention and neglect. Trends Cogn. Sci. (Regul. Ed.) 3 , 385-393. doi:10.1016/S13646613(99)01378-9

Rorden, C., and Karnath, H. O. (2010). A simple measure of neglect severity. Neuropsychologia 48, 2758-2763. doi:10.1016/j.neuropsychologia. 2010.04.018

Rossetti, Y., Rode, G., Pisella, L., Farne, A., Li, L., and Boisson, D. (1999). "Sensorimotor plasticity and cognition; prism adaptation can affect various levels of space representation," in Studies in Perception and Action, eds M. Grealy, and J. A. Thomson (Hove: Lawrence Erlbaum Associates), 265-269.

Rossetti, Y., Rode, G., Pisella, L., Farne, A., Li, L., Boisson, D., et al. (1998). Prism adaptation to a rightward optical deviation rehabilitates left hemispatial neglect. Nature 395, 166-169. doi:10.1038/25988

Saevarsson, S., Kristjansson, A., and Halsband, U. (2010). Strength in numbers: combining neck vibration and prism adaptation produces additive therapeutic effects in unilateral neglect. $\mathrm{Neu}$ ropsychol. Rehabil. 20, 704-724. doi:10.1080/09602011003737087

Saj, A., and Vuilleumier, P. (2007). Neglect: remembering the space left behind. Curr. Biol. 17, R1060-R1062. doi:10.1016/j.cub.2007.10.016

Sarri, M., Greenwood, R., Kalra, L., and Driver, J. (2011). Prism adaptation does not change the rightward spatial preference bias found with ambiguous stimuli in unilateral neglect. Cortex 47, 353-366. doi:10.1016/j.cortex.2010.01.006

Schatz, P., and Browndyke, J. (2002). Applications of computer-based neuropsychological assessment. J. Head Trauma Rehabil. 17, 395-410. doi:10.1097/00001199-20021000000003

Schenk, T., and Karnath, H. O. (2012). Neglect and attention: current trends and questions. Neuropsychologia 50, 1007-1009. doi:10.1016/j.neuropsychologia. 2012.03.029

Serino, A., Barbiani, M., Rinaldesi, M. L., and Làdavas, E. (2009). Effectiveness of prism adaptation in neglect rehabilitation: a controlled trial study. Stroke 40, 1392-1398. doi:10. 1161/STROKEAHA.108.530485

Striemer, C. L., and Danckert, J. (2010). Dissociating perceptual and motor effects of prism adaptation in neglect. Neuroreport 21, 436-441. doi:10.1097/WNR.0b013e328338 $592 \mathrm{f}$

Tilikete, C., Rode, G., Rossetti, Y. Pichon, J., Li, L., and Boisson, D. (2001). Prism adaptation to rightward optical deviation improves postural imbalance in left-hemiparetic patients. Curr. Biol. 11, 524-528. doi:10.1016/S09609822(01)00151-8

Tsirlin, I., Dupierrix, E., Chokron, S., Coquillart, S., and Ohlmann, T. (2009). Uses of virtual reality for diagnosis, rehabilitation and study of unilateral spatial neglect: review and analysis. Cyberpsychol. Behav. 12, 175-181. doi: $10.1089 / \mathrm{cpb} .2008 .0208$

Turton, A. J., O’Leary, K., Gabb, J., Woodward, R., and Gilchrist, I. D. (2010). A single blinded randomised controlled pilot trial of prism adaptation for improving self-care in stroke patients with neglect. Neuropsychol. Rehabil. 20, 180-196. doi:10.1080/09602010903040683

Watanabe, S., and Amimoto, K. (2010). Generalization of prism adaptation for wheelchair driving task in patients with unilateral spatial neglect. Arch. Phys. Med. Rehabil. 91, 443-447. doi:10.1016/j.apmr.2009.09.027

Conflict of Interest Statement: The authors declare that the research was conducted in the absence of any commercial or financial relationships that could be construed as a potential conflict of interest.

Received: 01 March 2013; accepted: 19 June 2013; published online: 24 July 2013. Citation: Smit M, Van der Stigchel $S$, Visser-Meily JMA, Kouwenhoven M, Eijsackers ALH and Nijboer TCW (2013) The feasibility of computer-based prism adaptation to ameliorate neglect in subacute stroke patients admitted to a rehabilitation center. Front. Hum. Neurosci. 7:353. doi: 10.3389/fnhum.2013.00353 Copyright (c) 2013 Smit, Van der Stigchel, Visser-Meily, Kouwenhoven, Eijsackers and Nijboer. This is an open-access article distributed under the terms of the Creative Commons Attribution License, which permits use, distribution and reproduction in other forums, provided the original authors and source are credited and subject to any copyright notices concerning any third-party graphics etc. 